\title{
Inhibition of Hyperhomocysteinemia- Induced Inflammasome Activation and Glomerular Sclerosis by NLRP3 Gene Deletion
}

\author{
Min Xia Sabena M. Conley Guangbi Li Pin-Lan Li Krishna M. Boini \\ Department of Pharmacology and Toxicology, Virginia Commonwealth University, School of Medicine, \\ Richmond, VA, USA
}

\section{Key Words}

hHcys $・ \mathrm{NLRP3} \cdot$ Inflammasomes $•$ Glomerulosclerosis $・$ End-stage renal disease

\begin{abstract}
Background/Aims: Hyperhomocysteinemia (hHcys) has been reported to initiate Nod-like receptor protein 3 (NLRP3) inflammasome formation and activation in podocytes, leading to glomerular dysfunction and sclerosis. However, it remains unknown whether Nlrp3 gene is critical for the formation and activation of inflammasomes in glomeruli of hHcys mice. Methods: Plasma homocysteine concentration was estimated utilizing HPLC, inflammasome formation and immunofluorescence expression from confocal microscopy, IL-1 $\beta$ production

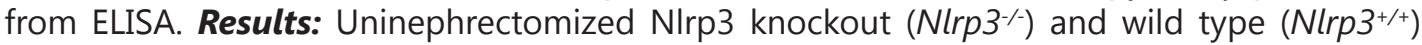
and intra renal NIrp3 shRNA-transfected wild type mice (NIrp3 shRNA) were fed a folate free (FF) diet or normal chow (ND) for 4 weeks to produce hHcys. The plasma Hcys levels were significantly elevated in both $\mathrm{Nlrp3}^{\%}$ and $\mathrm{Nlrp3}^{+/+}$mice fed a FF diet compared to ND fed mice. The FF diet significantly increased the colocalization of NIrp3 with apoptosis-associated speck-like protein (ASC) or caspase-1, caspase- 1 activity and IL-1 $\beta$ production in glomeruli of $\mathrm{Nlrp3}^{+/+}$, but not in Nlrp3 $3^{--}$mice and local Nlrp3 shRNA transfected mice. Correspondingly, the glomerular damage index (GDI) and urinary protein excretion were significantly higher in $\mathrm{Nlrp}^{+/+}$mice compared to ND fed mice. However, the hHcys-induced increase in GDI and proteinuria were significantly lower in NIrp3 ${ }^{-1}$ and local Nlrp3 shRNA transfected mice than in $\mathrm{Nlrp}^{+/+}$mice. Immunocytochemical analysis showed that hHcys decreased expression of podocin and nephrin, but increased desmin expression in glomeruli of $\mathrm{Nlrp3}^{+/+}$mice compared to Nlrp3\% mice. Conclusion: Nlrp3 gene is an essential component of Nlrp3 inflammasomes and that targeting Nlrp3 may be important therapeutic strategy to prevent inflammasome activation and thereby protect podocytes and glomeruli from hHcys-induced injury
\end{abstract}

Copyright (c) 2014 S. Karger AG, Basel 


\section{Cellular Physiology and Biochemistry}

Cell Physiol Biochem 2014;34:829-841

DOI: 10.1159/000363046

onlIne: August 20, 2014

C) 2014 S. Karger AG, Basel

www.karger.com/cpb

830

Xia et al.: NIrp3 in hHcys-Induced Inflammasome Activation

\section{Introduction}

Hyperhomocysteinemia (hHcys) is a pathological condition characterized by an increase in plasma concentration of total homocysteine [1-6]. Numerous clinical and epidemiological studies have demonstrated that hHcys is an independent risk factor for many diseases and pathological processes, including neurological, cardiovascular, and end stage renal diseases [7-9]. Recently we and others have demonstrated that hHcys initiates glomerular damage by inducing NADPH oxidase mediated local oxidative stress, ceramide production, podocyte injury and inhibition of extracellular matrix degradation contributing to the sclerotic process and eventually leading to the loss of kidney function $[1,2,5,10-13]$. In addition, we have also demonstrated that chronic elevations of plasma Hcys levels importantly contribute to the development of glomerular disease independent of hypertension in hHcys mice and rat models $[13,14]$. Despite extensive studies, the early mechanisms triggering the pathogenic actions of hHcys are not fully understood.

In this regard, the inflammasome is considered as an intracellular machinery of inflammation [15]. Among different kinds of inflammasomes, the Nlrp3 inflammasome is the most fully characterized one in a variety of mammalian cells with high abundance in glomerular podocytes $[13,16,17]$. The Nlrp3 inflammasome is composed of three major proteins, including a NOD-like receptor Nlrp3, an adaptor protein apoptosis-associated speck-like protein containing a caspase recruitment domain (ASC), and caspase-1 [18-21]. Nlrp3 acts as the sensory component to recognize both endogenous and exogenous danger signals [22-24], when ASC and caspase-1 are recruited to form a protein complex, where caspase- 1 is activated. The active caspase- 1 not only proteolytically cleaves IL- $1 \beta$ and/or IL-18 into their biologically active form, but also produces other deleterious molecules like damage-associated molecular patterns (DAMPs). Both will act to turn on inflammatory response and induce cell dysfunction. This multiprotein complex was first characterized in monocytes [25, 26], and its abnormality was attributed to the development of several autoinflammatory disorders including Familial cold autoinflammatory syndrome, MuckleWells syndrome and Sweet's syndrome [18, 27, 28]. There is now increasing evidence that the derangement of this intracellular inflammatory machinery may not only contribute to autoinflammatory disorders, but also to a number of metabolic diseases including gout, silicosis, diabetes, acetaminophen liver toxicity, Alzheimer's disease and atherosclerosis [21, 29-36]. Correspondingly, the Nlrp3 inflammasome was found to be stimulated by various endogenous stress-associated danger signals such as DAMPs [37, 38], ATP [39], monosodium urate crystals [31], obesity [40], $\beta$-amyloid [23], acute ischemia/reperfusioninduced kidney injury [41], unilateral ureteral obstruction [16, 17]. Interestingly, inhibition of inflammasomes by either ASC gene silencing or pharmacological inhibition of casapase- 1 has recently been shown to prevent the development of hHcys-induced glomerular injury and podocyte injury [13]. However, it remains unknown whether Nlrp3 gene is critical for the formation and activation of inflammasomes in glomeruli of hHcys mice

The present study hypothesized that Nlrp3 gene deficiency attenuates the glomeruli from hHcys-induced inflammasome formation and thereby ameliorate glomerulosclerosis and podocyte injury under such pathological condition. To test this hypothesis, we first performed a series of experiments using $\mathrm{Nlrp}^{\%}$ and their wild type littermates on the normal chow or folate free diet to determine whether lack of Nlrp3 gene alters the formation and activation of Nlrp3 inflammasomes, glomerular injury and podocyte injury in mice during hHcys. Then, we locally silenced renal Nlrp3 gene using shRNA and observed the effects of renal Nlrp3 deficiency on hHcys-induced glomerular inflammasome formation and corresponding injury. Our results demonstrate that Nlrp3 gene is an essential component of Nlrp3 inflammasomes and that targeting Nlrp3 may be important therapeutic strategy to prevent inflammasome formation and activation and thereby protect podocytes and glomeruli from hHcys-induced injury 


\section{Cellular Physiology and Biochemistry}

Cell Physiol Biochem 2014;34:829-841

\begin{tabular}{l|l}
\hline DOI: $10.1159 / 000363046$ & (C) 2014 S. Karger AG, Basel
\end{tabular}

www.karger.com/cpb

Xia et al.: NIrp3 in hHcys-Induced Inflammasome Activation

\section{Materials and Methods}

Animals

Eight weeks old male C57BL/6J WT, Nlrp3\% mice and their wild type littermates were used in the present study. To speed up the damaging effects of hHcys on glomeruli, all mice were uninephrectomized as we described previously $[1,2]$. This model has been demonstrated to induce glomerular damage unrelated to the uninephrectomy and arterial blood pressure, but specific to hHcys. After a 1-week recovery period from uninephrectomy, mice were fed either normal chow or folate-free (FF) diet (Dyets Inc, Bethlehem, PA, USA) for 4 weeks to induce hHcys. In another series of C57BL/6J mice, Nlrp3 shRNA or a scrambled shRNA (Origen, Rockville, MD, USA) plasmid with a luciferase expression vector was co-transfected into the kidneys via intrarenal artery injection using the ultrasound microbubble system as we described previously $[2,13,42]$. After delivery of plasmids into the kidney, these uninephrectomized mice were maintained on a normal or a FF diet for 4 weeks. All protocols were approved by the Institutional Animal Care and Use Committee of the Virginia Commonwealth University.

\section{High-performance liquid chromatography (HPLC) analysis}

Plasma and renal tissue Hcys levels were measured by HPLC method as we described previously [1, 43, 44]. A $100 \mu \mathrm{L}$ plasma or samples or standard solution mixed with $10 \mu \mathrm{L}$ of internal standard, thionglycolic acid $(2.0 \mathrm{mmol} / \mathrm{L})$ solution, was treated with $10 \mu \mathrm{L}$ of $10 \%$ tri-n-butylphosphine (TBP) solution in dimethylformamide at $4{ }^{\circ} \mathrm{C}$ for 30 minutes. Then, $80 \mu \mathrm{L}$ of ice-cold $10 \%$ trichloro acetic acid (TCA) in 1 $\mathrm{mmol} / \mathrm{L}$ EDTA was added and centrifuged to remove proteins in the sample. $100 \mu \mathrm{L}$ of the supernatant was transferred into the mixture of $20 \mu \mathrm{L}$ of $1.55 \mathrm{~mol} / \mathrm{L}$ sodium hydroxide, $250 \mu \mathrm{L}$ of $0.125 \mathrm{~mol} / \mathrm{L}$ borate buffer ( $\mathrm{pH}$ 9.5), and $100 \mu \mathrm{L}$ of $1.0 \mathrm{mg} / \mathrm{mL}$ ABD-F solution. The resulting mixture was incubated at $60^{\circ} \mathrm{C}$ for 30 minutes to accomplish derivatization of thiols. HPLC was performed with a HP 1100 series equipped with a binary pump, a vacuum degasser, a thermo stated column compartment, and an auto sampler (Agilent Technologies, Waldbronn, Germany). Separation was carried out at an ambient temperature on an analytical column, Supelco LC-18-DB (1504.6 mm ID, 5 m) with a Supelcosil LC-18 guard column (204.6 mm ID, $5 \mathrm{~m}$ ). Fluorescence intensities were measured with an excitation wavelength of $385 \mathrm{~nm}$ and emission wavelength of $515 \mathrm{~nm}$ by a Hewlett-Packard Model 1046A fluorescence spectrophotometer. The peak area of the chromatographs was quantified with a Hewlett-Packard 3392 integrator. The analytical column was eluted with $0.1 \mathrm{~mol} / \mathrm{L}$ potassium dihydrogen phosphate buffer ( $\mathrm{pH} 2.1)$ containing $6 \%$ acetonitrile $(\mathrm{v} / \mathrm{v})$ as the mobile phase with a flow rate of $2.0 \mathrm{~mL} / \mathrm{min}$.

\section{Caspase- 1 activity and $I L-1 \beta$ production}

Caspase-1 activity in glomeruli was measured by a commercially available colorimetric assay kit (Biovision, Mountain View, CA). IL-1 $\beta$ production in glomeruli was measured by a commercially available ELISA kit (R\&D System, Minneapolis, MN), according to the manufacturer's instructions $[11,13,40]$.

\section{Morphological examinations}

The fixed kidneys were paraffin-embedded, and sections were prepared and stained with Periodic acid-Schiff stain. Glomerular damage index (GDI) was calculated from 0 to 4 on the basis of the degree of glomerulosclerosis and mesangial matrix expansion as described previously [40, 42, 45]. In general, we counted 50 glomeruli in total in each kidney slice under microscope, when each glomerulus was graded level 0-4 damages. 0 represents no lesion, $1+$ represents sclerosis of $<25 \%$ of the glomerulus, while $2+$, $3+$, and $4+$ represent sclerosis of $25 \%$ to $50 \%,>50 \%$ to $75 \%$, and $>75 \%$ of the glomerulus. A whole kidney average sclerosis index was obtained by averaging scores from counted glomeruli. This observation was examined by two independent investigators who were blinded to the treatment of the experimental groups $[2,11,13,42,45]$.

Urinary total protein and albumin excretion measurements:

The 24-hour urine samples were collected using metabolic cages and subjected to total protein and albumin excretion measurements, respectively $[2,42,45,46]$. Total protein content in the urine was detected by Bradford method using a UV spectrophotometer. Urine albumin was detected using a commercially available albumin ELISA kit (Bethyl Laboratories, Montgomery, TX). 


\section{Cellular Physiology and Biochemistry}

Cell Physiol Biochem 2014;34:829-841

DOI: $10.1159 / 000363046$

Publisned oniline: August 20, 2014

c) 2014 S. Karger AG, Basel

www.karger.com/cpb

Xia et al.: Nlrp3 in hHcys-Induced Inflammasome Activation

Delivery of Nlrp3 shRNA into the kidneys by ultrasound-microbubble technique

Nlrp3 shRNA or a scrambled shRNA plasmid with a luciferase expression vector was used to cotransfect the kidneys via intrarenal artery injection using the ultrasound-microbubble system. These experiments were performed to test whether local silencing Nlrp3 gene expression in podocytes alters hHcys-induced glomerular injury. A full description of the procedures for the ultrasound-microbubble gene transfer technique can be found in our previous studies $[2,13,42]$. To monitor the efficiency of gene expression through somatic plasmid transfection daily, mice were anesthetized with isoflurane, and an aqueous solution of luciferin (150 mg/kg) was injected intraperitoneally 5 minutes before imaging. The anesthetized mice were imaged using the IVIS200 in vivo molecular imaging system (Xenogen, Hopkinton, MA, USA). Photons emitted from luciferase-expressing cells within the animal body and transmitted through tissue layers were quantified over a defined period of time ranging up to 5 minutes using the software program Living Image as program (Xenogen). The inhibitory efficiency of gene expression by Nlrp3 shRNA was further confirmed by detection of Nlrp3 level in mouse renal cortex using real-time RT-PCR and Immunofluorescence analysis.

\section{Real-time reverse transcription polymerase chain reaction (RT-PCR)}

Total RNA from isolated mouse renal tissue was extracted using TRIzol reagent (Invitrogen, Carlsbad, CA) according to the protocol as described by the manufacturer. RNA samples were quantified by measurement of optic absorbance at $260 \mathrm{~nm}$ and $280 \mathrm{~nm}$ in a spectrophotometer. The concentrations of RNA were calculated according to A260. Aliquots of total RNA (1 $\mu \mathrm{g})$ from each sample were reverse-transcribed into cDNA according to the instructions of the first strand cDNA synthesis kit manufacturer (Bio-Rad, Hercules, CA). Equal amounts of the reverse transcriptional products were subjected to PCR amplification using SYBR Green as the fluorescence indicator on a Bio-Rad iCycler system (Bio-Rad, Hercules, CA) [2, 13, 42]. The primers used in this study were synthesized by Operon (Huntsville, AL, USA) and the sequences were: for Nlrp3 sense TCACAACTCGCCCAAGGAGGAA, antisense AAGAGACCACGGCAGA-AGCTAG and for $\beta$-actin sense TCGCTGCGCTGGTCGTC, antisense GGCCTCGTCACCCACATAGGA.

\section{Confocal microscopic detection of inflammasome protein complexes}

Indirect immunofluorescent staining was used to determine colocalization of the inflammasome proteins in glomeruli of the mouse kidney, which indicate the formation of inflammasome molecular complex. Frozen kidney tissue slides were fixed in acetone and then incubated overnight at $4^{\circ} \mathrm{C}$ with either goat antiNalp3 (1:200) and rabbit anti-Asc (1:50), or goat anti- Nalp3 (1:200) and mouse anti-caspase-1 (1:100). To further confirm the presence of the inflammasomes specifically in podocytes of the mouse glomeruli, Nalp3 was co incubated with a podocin antibody (1:400; Sigma, St. Louis, MO). Double immunofluorescent staining was achieved by incubating with either Alexa-488 or Alexa-555-labeled secondary antibodies for 1 hour at room temperature. After washing, slides were mounted with a DAPI-containing mounting solution, and then observed with a confocal laser scanning microscope (Fluoview FV1000, Olympus, Japan). As previously described $[13,42]$, images were analyzed by the Image Pro Plus 6.0 software (Media Cybernetics, Bethesda, MD), where colocalization was measured and expressed as the Pearson Correlation Coefficient (PCC).

\section{Immunofluorescent staining}

Immunofluorescent staining was performed using frozen slides of mouse kidneys. After fixation with acetone, the slides were incubated with anti-podocin (Sigma, St. Louis, MO, USA, 1: 100), anti-desmin (BD Biosciences, San Jose, CA, 1: 50), anti-nephrin (Abcam, Cambridge, MA, USA, 1:50) antibodies overnight at $4^{\circ} \mathrm{C}$. Then, these slides were washed and incubated with corresponding Texas Red-labeled secondary antibodies. Finally, the slides were washed, mounted and subjected to fluorescent microscopy examination. The images were captured with a spot CCD camera and a pseudocolor was added to fluorescence showing in this slide (Diagnostic Instruments Inc., Sterlin Heights, MI, USA). All exposure settings were kept constant for each group of kidneys.

\section{Immunohistochemistry}

Kidneys were embedded with paraffin and $5 \mathrm{~mm}$ sections were cut from the embedded blocks. After heat-induced antigen retrieval, wash with $3 \% \mathrm{H}_{2} \mathrm{O}_{2}$ and 30 min blocking with serum, slides were 


\section{Cellular Physiology and Biochemistry}

Cell Physiol Biochem 2014;34:829-841

Fig. 1. hHcys-induced Nlrp3 inflammasome formation in glomeruli of $\mathrm{Nlrp3}^{+/+}$and $\mathrm{Nlrp3}^{\% /-}$ mice fed a normal diet or folate free diet. A: Genotyping, B. Colocalization of Nlrp3 (green) with ASC (red), Nlrp3 (green) with caspase-1 (red) and Nlrp3 (green) with podocin (red) in mouse glomeruli. C. Summarized data shows the fold changes of pearson coefficient correlation (PCC) for the colocalization of Nlrp3 with ASC and Nlrp3 with caspase-1 in glomeruli of Nlrp3+/+ and Nlrp3-/- mice fed with ND or FF diet. * Significant difference $(P<0.05)$ compared to the values from control Nlrp3 ${ }^{+/+}$mice, ${ }^{*}$ Significant difference $(P<0.05)$ compared to the values from $\mathrm{Nlrp3}^{+/+}$ mice receiving the FF diet.

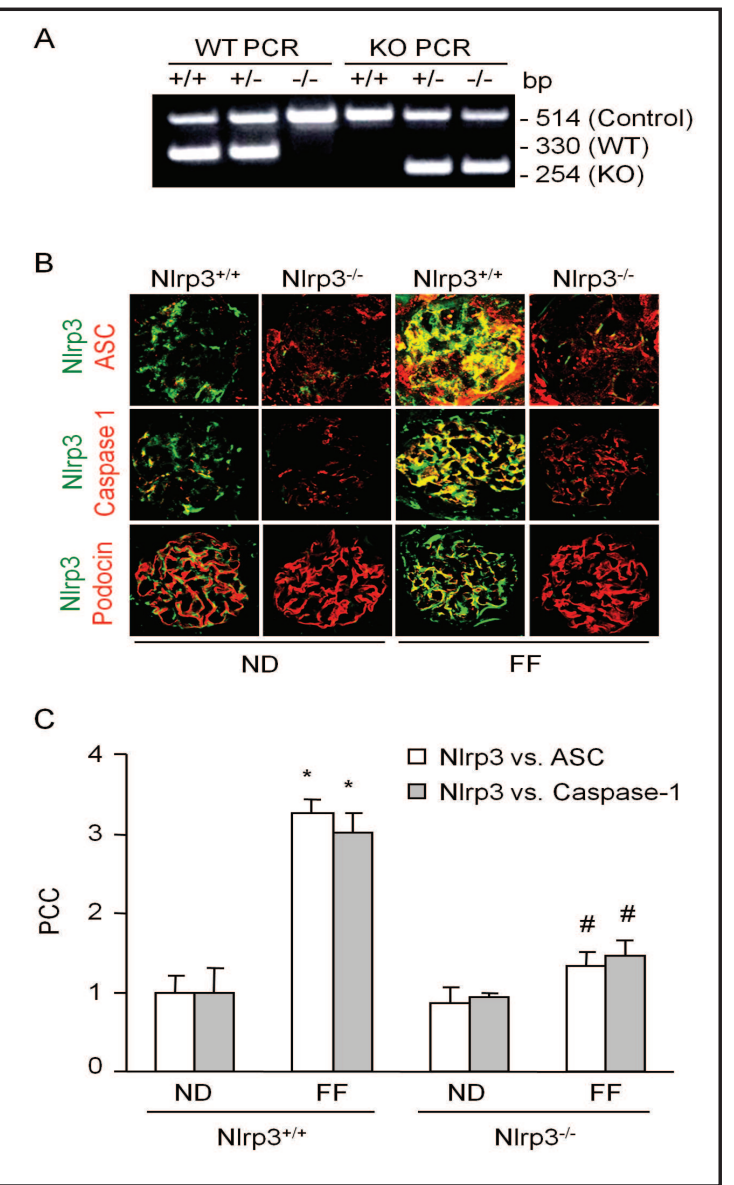

incubated with primary antibody diluted in phosphate-buffered saline (PBS) with $4 \%$ serum. Anti-IL-1 $\beta$ (Abcam, Cambridge, MA, USA) antibody was used in this study. After incubation with primary antibody overnight, the sections were washed in PBS and incubated with biotinylated IgG (1:200) for $1 \mathrm{~h}$ and then with streptavidin-HRP for $30 \mathrm{~min}$ at room temperature. $50 \mu \mathrm{l}$ of DAB was added to each kidney section and stained for $1 \mathrm{~min}$. After washing, the slides were counterstained with hematoxylin for $5 \mathrm{~min}$. The slides were then mounted and observed under a microscope in which photos were taken.

\section{Statistical Analysis}

Data are provided as arithmetic means \pm SEM; $n$ represents the number of independent experiments. All data were tested for significance using ANOVA or paired and unpaired Student's t-test as applicable. The glomerular damage index was analysed using a nonparametric Mann-Whitney rank sum test. Only results with $\mathrm{p}<0.05$ were considered statistically significant.

\section{Results}

Nlrp3 gene deletion abolished hHcys-induced inflammasome formation and activation in glomeruli

The plasma Hcys concentration was similar in $\mathrm{Nlrp}^{+/+}$and Nlrp3 ${ }^{-/-}$mice fed a normal diet $\left(\mathrm{Nlrp3}^{+/+}: 4.9 \pm 0.5\right.$ vs Nlrp3\% $\left.7.0 \pm 0.6\right)$. However, the FF diet significantly increased the plasma Hcys concentration in both mouse strains compared to the normal diet fed mice (Nlrp3 $3^{+/+}: 39.5 \pm$ 12 vs Nlrp3\%/: $44 \pm 7$ ). As shown in Fig. 1A, Nlrp3 knockout (Nlrp3/-) and wild type (Nlrp3 ${ }^{+/+}$) mice were genotyped using PCR. Detection of a PCR product at $254 \mathrm{bp}$ indicates Nlrp3\% , while a PCR product of $330 \mathrm{bp}$ indicates Nlrp3 $3^{+/}$mice. Next, we tested whether Nlrp3 mediates the hHcys-induced inflammasome formation and activation in glomeruli of mice. Using 
Fig. 2. Effects of normal diet and folate free diet on caspase- 1 activity and IL-1 $\beta$ production in $\mathrm{Nlrp}^{+/+}$and Nlrp3\% mice. Values are arithmetic means \pm SEM ( $n=6$ each group) of caspase-1 activity (A), IL-1B production (B-C) in glomeruli of $\mathrm{Nlrp3}^{+/+}$ and $\mathrm{Nlrp}^{-\%}$ mice fed with ND or FF diet. Significant difference $(P<0.05)$ compared to the values from control $\mathrm{Nlrp3}^{+/+}$mice, \# Significant difference $(P<0.05)$ compared to the values from $\mathrm{Nlrp3}^{+/+}$mice receiving the FF diet.

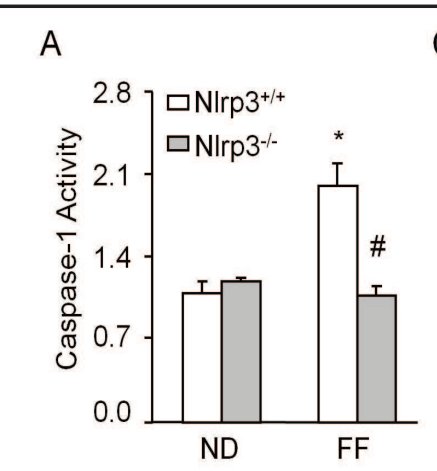

C

\section{B}

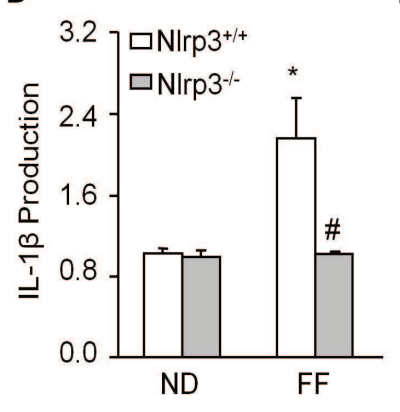

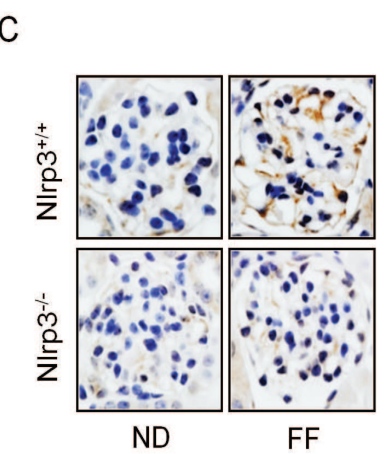

D

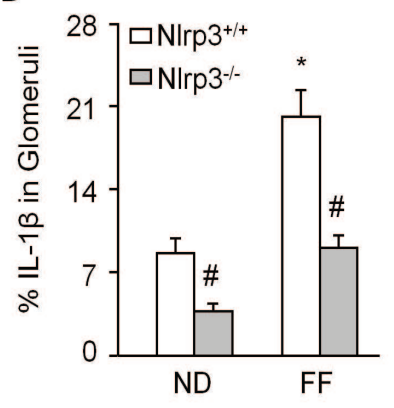

confocal microscopy, we demonstrated that hHcys increased the colocalization of Nlrp3 with ASC or Nlrp3 with caspase-1 in glomeruli of mice compared to normal diet fed mice. However, $\mathrm{Nlrp}^{-/}$mice attenuated the hHcys-induced colocalization of Nlrp3 with ASC or Nlrp3 with caspase-1 in glomeruli of mice (Fig. 1B). Furthermore, colocalization of Nlrp3 with podocin (podocyte marker) indicates enrichment of Nlrp3 in podocytes. The summarized data of quantitative colocalization of Nlrp3 with Asc or Nlrp3 with caspase-1 in glomeruli of mice were shown in Fig. 1C. Biochemical analysis showed that hHcys significantly increased the caspase activity and IL-1 $\beta$ production in glomeruli of $\mathrm{Nlrp}^{+/+}$mice. However, Nlrp3 ${ }^{-/}$mice attenuated the hHcys-induced caspase- 1 activity and IL-1 $\beta$ production (Fig. 2). Taken together, these results suggest that Nlrp3 mediates the hHcysinduced NLRP3 inflammasome formation and activation in glomeruli of mice.

Mice lacking Nlrp3 gene protects against hHcys-induced glomerular injury and podocyte injury

As shown in Figure 3A, urinary albumin excretion were similar in Nlrp3 $3^{+/+}$and $\mathrm{Nlrp} 3^{-/}$ mice fed a normal diet. However, hHcys significantly increased the urinary albumin excretion in $\mathrm{Nlrp}^{+/+}$mice, but not in Nlrp3 $\%$ mice. By PAS staining, we observed a typical pathological change in glomerular sclerotic damage in $\mathrm{Nlrp}^{+/+}$mice on the folate free diet such as glomerular capillary collapse and mesangial expansion. This pathology was not observed in Nlrp3\% mice. The glomerular damage index (GDI) was significantly higher in $\mathrm{Nlrp}^{+/+}$mice fed a FF diet compared to ND fed mice. However, the hHcys-induced glomerular damage index was significantly attenuated in Nlrp3 $\%$ mice (Fig. 3B and 3C). Further experiments were performed to test whether inflammasome-mediated glomerular injury is due to podocyte dysfunction or injury. By immunofluorescent microscopic analysis, desmin was found to have more profound abundance in glomeruli of FF diet-fed mice compared to that in normal diet-fed mice. The lack of Nlrp3 gene abrogated the FF diet-induced increase in desmin expression (Figure 4A). In contrast, nephrin and podocin were markedly reduced in glomeruli from FF diet-fed mice compared to those in normal diet-fed mice. However, the hHcys $\mathrm{Nlrp}^{-/}$mice completely attenuated the decrease in nephrin and podocin staining (Figure 4B and 4C). 


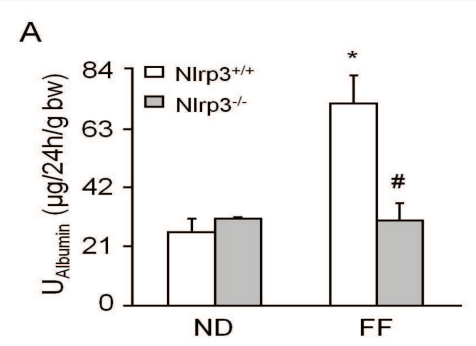

B

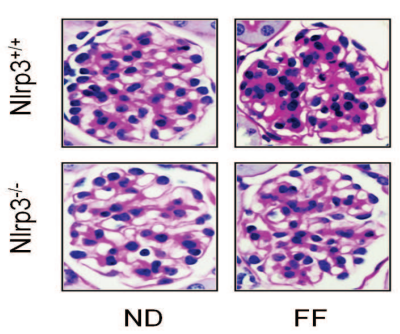

C

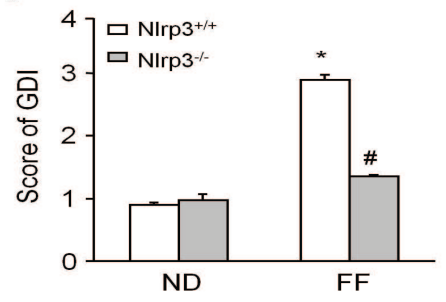

Fig. 3. Effects of the normal diet and FF Diet on glomerular injury in $\mathrm{Nlrp}^{+/+}$and $\mathrm{Nlrp} 3^{-/-}$mice. Values are arithmetic means \pm SEM ( $n=6$ each group) of urinary albumin (A) excretion in $\mathrm{Nlrp}^{+/+}$and Nlrp3 ${ }^{-1}$ mice with or without the FF diet. B: Photomicrographs show typical glomerular structure (original magnification, $\mathrm{x} 400$ ) in $\mathrm{Nlrp}^{+/+}$and Nlrp3 $\%$ mice fed with or without FF diet, C: Summarized data of glomerular damage index (GDI) by semi-quantitation of scores in 4 different groups of mice ( $n=6$ each group). For each kidney section, 50 glomeruli were randomly chosen for the calculation of GDI. Significant difference $(P<0.05)$ compared to the values from control Nlrp3 $3^{+/+}$mice, \# Significant difference $(P<0.05)$ compared to the values from $\mathrm{Nlrp3}^{+/+}$mice receiving the FF diet.

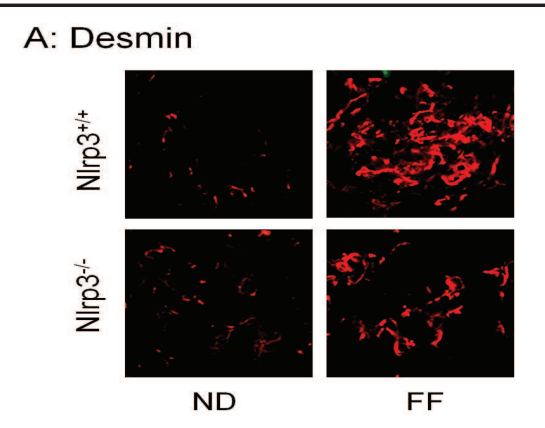

\section{B: Nephrin}
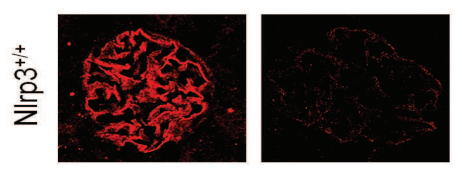

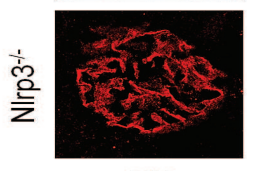

ND

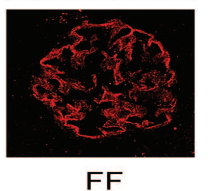

C: Podocin

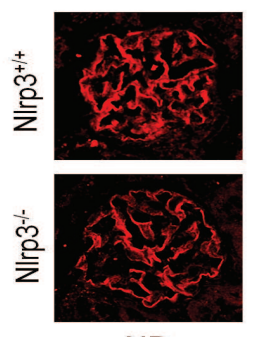

ND
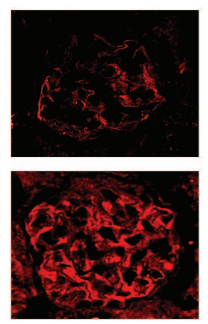

FF
Fig. 4. Immunofluorescence staining and expression of desmin, nephrin and podocin in glomeruli from Nlrp $3 \%$ and $\mathrm{Nlrp}^{3+/+}$ mice fed a ND or folate free diet. A. Typical images of desmin (A), nephrin (B) and podocin (C) staining in glomeruli from $\mathrm{Nlrp}^{\%} \%$ and $\mathrm{Nlrp}^{+/+}$mice with or without folate free diet.

\section{Efficiency of in vivo local transfection of Nlrp3 shRNA into the kidney}

As shown in Figure 5A, we used an IVIS in vivo molecular imaging system to detect the expression of co-transfected luciferase gene, which insures an efficient delivery of target gene into the mouse kidney. The luciferase reporter gene was monitored in to the kidney of the living mouse after the injection of plasmid mixed with microbubbles under ultrasound force. Starting on day 3, the expression of luciferase gene persisted for 4 weeks. In the hemidissected kidney, all of the cortical regions were observed to exhibit efficient gene transfection as shown in green and red fluorescence (Fig. 5B). As illustrated in Fig. 5C and 5D, Nlrp3 expression was significantly decreased in C57BL/6J WT mice transfected with Nlrp3 shRNA compared to control mice (scramble shRNA) fed a normal diet. Compared to the normal diet fed mice, the hHcys 
Fig. 5. Effects of local Nlrp3 gene silencing on hHcys-induced Nlrp3 inflammasome formation and activation in C57BL/6J mice with or without the FF diet. Nlrp3 shRNA (Nlrp3 sh) and luciferase cDNA plasmids were locally delivered into the kidney. A: Daily imaging confirmation of gene transfection by detection of cotransfected luciferase gene expression by using an IVIS in vivo molecular imaging system. B: Localization of luciferase gene expression in hemidissected kidney on day 12 after gene delivery. C: Nlrp3 Immunofluorescent staining, D: Values are arithmetic means \pm SEM $(n=5-6$ each group) of Nlrp3 mRNA expression in C57BL/6 mice on the normal or folate free diet with or without Nlrp3 shRNA transfection. Scra:

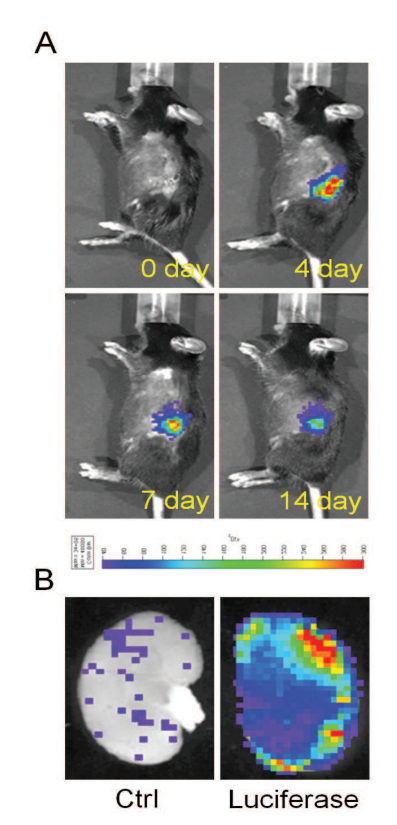

C

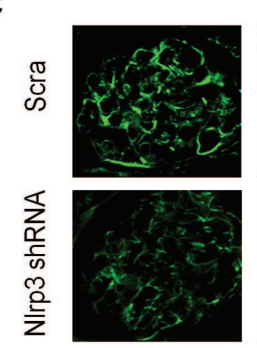

ND

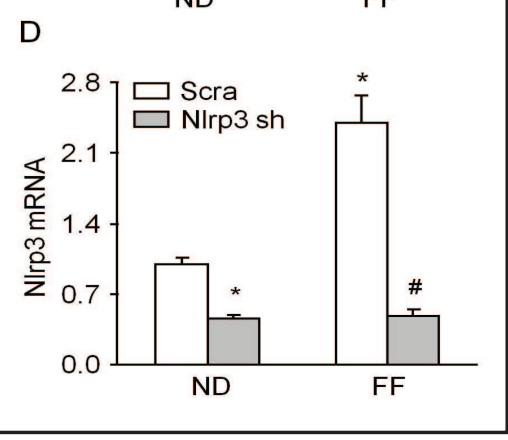

Scramble, Nlrp3 sh: Nlrp3 shRNA, *Significant difference $(P<0.05)$ compared to the values from control mice on the normal diet, " Significant difference $(P<0.05)$ compared to the values from mice on the folate free diet.

significantly increased the Nlrp3 expression in glomeruli from mice receiving scrambled shRNA, but it had no effect on in mice receiving Nlrp3 shRNA.

Attenuation of hHcys-induced inflammasome formation, glomerular injury by Nlrp3 gene silencing

Further we determined whether Nlrp3 gene silencing locally in the kidney may attenuate the hHcys-induced Nlrp3 inflammasome formation and protects against the glomerular injury. As shown in Figure 6A, hHcys increased the colocalization of Nlrp3 with caspase-1 in glomeruli of scrambled shRNA transfected mice. However, such colocalization was not observed in glomeruli of Nlrp3 shRNA transfected mice, suggesting the attenuation of inflammasome formation in glomeruli. In consistent with the decreased Nlrp3 inflammasome formation, caspase- 1 activity was attenuated in Nlrp3 shRNA transfected mice (Fig. 6B). The urinary protein excretion was similar in both scrambled and Nlrp3 shRNA transfected mice fed a normal diet. hHcys significantly increased the urinary total protein excretion when compared to the normal diet-fed mice. However, the Nlrp3 shRNA transfection significantly attenuated hHcys-induced urinary total protein excretion (Fig. 6C).

\section{Discussion}

The major goal of the present study is to determine the role Nlrp3 gene in the formation and activation of NLRP3 inflammasomes in glomeruli of hHcys mice. We found that hHcys-induced the Nlrp3 inflammasome formation and activation and contributes to the podocyte injury and glomerular injury/sclerosis in $\mathrm{Nlrp}^{+/+}$mice. The deficiency of Nlrp3 gene completely abolished the hHcys-induced inflammasome formation, glomerular injury and podocyte injury. Our results for the first time demonstrate that NLRP3 gene is a critical component in mediating the hHcys-induced NLRP3 inflammasome formation and glomerular injury. 
Fig. 6. Effects of renal Nlrp3 gene silencing on glomerular injury in C57BL/6J mice with or without the folate free diet. A: Colocalization of Nlrp3 (green) with caspase-1 (red) in mouse glomeruli, B: caspase- 1 activity, C: Values are arithmetic means \pm SEM ( $n=5-6$ each group) of urinary total protein excretion in C57BL/6J mice on the normal or folate free diet with or without Nlrp3 shRNA transfection. Scra: Scramble, Nlrp3 sh: Nlrp3 shRNA, "Significant difference $(P<0.05)$ compared to the values from control mice on the normal diet, " Significant difference $(P<0.05)$ compared to the values from mice on the folate free diet.

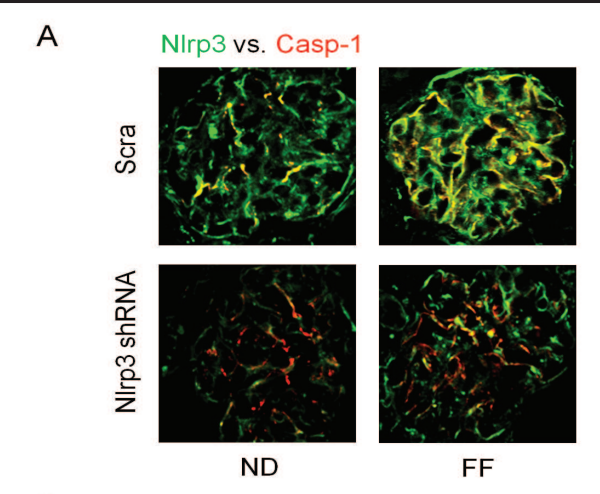

B

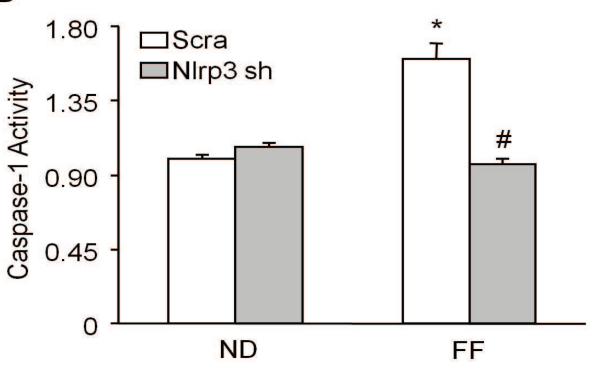

C

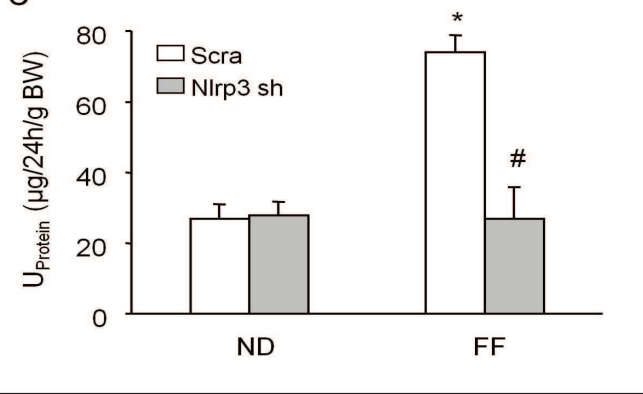

It has been reported that NLRP3 inflammasome, an inflammatory machinery that when stimulated results in IL-1 $\beta$ maturation and triggers the early innate immunity cascade to initiate an immune response, is comprised of three major proteins - NLRP3, considered to be the sensory component, the adaptor protein ASC, and the effector caspase-1. Deficiencies in this multiprotein complex have been associated with autoimmune disorders such as familial Mediterranean fever and Muckle-Wells syndrome, but aberrant NLRP3 inflammasome activation has extended to many traditionally considered non-inflammatory disorders including diabetes, obesity, silicosis, acute myocardial infarction, liver toxicity, and kidney diseases $[21,29,31,47-50]$. Most recently we have demonstrated that inhibition of NLRP3 inflammasomes either by apoptosis associated speck-like protein (ASC) gene silencing or by pharmacological inhibition of caspase- 1 attenuated the hHcys-induced glomerular injury [13]; however, the role of NLRP3 gene in hHcys-induced glomerular injury/sclerosis has not been elucidated. Therefore, we used Nlrp3 deficient mice to explore the role of Nlrp3 gene in mediating the hHcys-induced glomerular injury or sclerosis. It was found that FF diet treatment for 4 weeks significantly increased the plasma Hcys levels in both $\mathrm{Nlrp}^{+/+}$and Nlrp3 $\%$ mice, which indicates a successful establishment of the hHcys and also suggests that Nlrp3 gene is not involved in the global metabolism of Hcys. The increased plasma Hcys concentration induced the formation of NLRP3 inflammasomes in glomeruli as shown by colocalization of NLRP3 with ASC or NLRP3 with caspase in $\mathrm{Nlrp}^{+/+}$mice. Using podocin as a podocyte marker, our confocal observations demonstrated that hHcys-induced inflammasome formation in glomeruli was mostly located in podocytes as demonstrated by 


\section{Cellular Physiology and Biochemistry}

Cell Physiol Biochem 2014;34:829-841

\begin{tabular}{l|l}
\hline DOI: $10.1159 / 000363046$ & (C) 2014 S. Karger AG, Basel
\end{tabular}

www.karger.com/cpb

Xia et al.: NIrp3 in hHcys-Induced Inflammasome Activation

the colocalization of Nlrp3 with podocin. These colocalizations were substantially blocked in mice lacking Nlrp3 gene. Moreover, the biochemical analysis showed that hHcys increased the caspase- 1 activity and IL-1 $\beta$ production in Nlrp3 ${ }^{+/+}$mice but not in Nlrp $3^{-/}$mice, suggesting the essential role of NLRP3 gene in mediating the hHcys-induced inflammasome formation and activation in glomeruli of mice.

In previous studies, Nlrp3 inflammasome has been implicated in the regulation of kidney function $[16,17,41]$. Previous reports from our laboratory and others demonstrated the role of inflammasome in hHcys or obesity-induced glomerular injury $[11,13,40]$, acute ischemia/ reperfusion-induced kidney injury [41], unilateral ureteral obstruction [16, 17], and renal biopsies from patients with non-diabetic kidney disease [17]. Inhibition of inflammasomes attenuated the proteinuria and kidney function. In this study, we tested, whether NLRP3 gene is critically involved in the hHcys-induced glomerular injury. In accordance with decreased inflammasome formation in hHcys Nlrp3\% mice, urinary albumin excretion and glomerular injury/sclerosis were significantly blocked compared with $\mathrm{Nlrp}^{+/+}$mice on the FF diet suggesting that that inflammasome formation and activation are an important early mechanism responsible for glomerular injury in hHcys mice. In particular, activation of podocyte or glomerular inflammasomes is critical for the development of glomerular injury associated with hHcys. Furthermore, we explored the mechanism mediating the protective action of NLRP3 gene in glomerular injury, we observed the changes in podocyte function in both $\mathrm{Nlrp}^{+/+}$and Nlrp3 $3^{--}$mice, given that podocyte dysfunction or effacement is considered the initiating mechanism to produce glomerular injury or sclerosis, leading to proteinuria with possible progression to $\operatorname{ESRD}[36,51]$. The present study showed that podocin and nephrin protein were markedly decreased in FF diet-fed $\mathrm{Nlrp}^{+/+}$mice, but not in mice lacking Nlrp3. While another podocyte injury marker, desmin was increased in hHcys $\mathrm{Nlrp}^{+/+}$mice, which was abolished in Nlrp3 ${ }^{-/}$mice. These results support the view that podocyte inflammasomes as an intracellular inflammatory machinery or responder to endogenous danger factors could be a target of therapeutic strategy for hHcys-induced glomerular injury and podocyte injury.

To further explore the role of NLRP3 gene in mediating hHcys induced-inflammasome formation and glomerular injury, we locally modulated gene expression of Nlrp3 in mouse kidney and observed changes in NLRP3 inflammasomes colocalization in glomeruli of mice. To reach this goal, we combined an ultrasound technique and microbubble wrapping for introduction of plasmids into kidney. Several recent reports from our laboratory and by others have shown that the ultrasound-microbubble system is a very effective method to deliver plasmids into cells of different organs in vivo with a transfection rate over 90\% [5255]. Combination of the ultrasound technique and microbubble wrapping for introduction of plasmids greatly enhances transgene expression with a 300 -fold increase over naked DNA alone without toxicity [56,57]. The present study using in vivo molecular imaging demonstrated that plasmids (Nlrp3 shRNA plasmids co-transfected with luciferase cDNA plasmid) were successfully delivered into the kidney and co-transfected luciferase expression persisted for up to 4 weeks. In such local Nlrp3 gene silenced kidney, we found that mRNA expression of Nlrp3, colocalization of Nalp3 with caspase-1 in mouse glomeruli and caspase 1 activity were significantly decreased. It was also demonstrated that silencing the Nlrp3 gene in the kidney ameliorates proteinuria. These results from mice with local renal Nlrp3 gene silencing further support the conclusion above drawn from studies using Nlrp $3 \%$ mice that Nlrp3 inhibition or gene silencing abolishes hHcys-induced action of inflammasome and thereby protect the kidney from hHcys-induced glomerular injury. In conclusion, the present study demonstrated that Nlrp3 gene deficiency attenuates the hHcys-induced inflammasome formation, glomerular injury and podocyte injury. Therefore, targeting Nlrp3 may be an important therapeutic strategy to prevent inflammasome activation and thereby protect podocytes and glomeruli from hHcys-induced injury. 


\section{Cellular Physiology and Biochemistry}

Cell Physiol Biochem 2014;34:829-841

\begin{tabular}{l|l}
\hline DOI: $10.1159 / 000363046$ & (c) 2014 S. Karger AG, Basel
\end{tabular}

Xia et al.: Nlrp3 in hHcys-Induced Inflammasome Activation

\section{Acknowledgements}

This work was supported by grants DK54927, HL091464, HL57244 and HL075316 from the National Institutes of Health (to P.L.) and VCU's CTSA (UL1TR000058 from the National Center for Advancing Translational Sciences) and the CCTR Endowment Fund of Virginia Commonwealth University (to K.B.).

\section{References}

1 Boini KM, Xia M, Abais JM, Xu M, Li CX, Li PL: Acid sphingomyelinase gene knockout ameliorates hyperhomocysteinemic glomerular injury in mice lacking cystathionine-beta-synthase. PLoS One 2012;7:e45020.

-2 Boini KM, Xia M, Li C, Zhang C, Payne LP, Abais JM, Poklis JL, Hylemon PB, Li PL: Acid sphingomyelinase gene deficiency ameliorates the hyperhomocysteinemia-induced glomerular injury in mice. Am J Pathol 2011;179:2210-2219.

-3 Grenz A, Hermes M, Hammel P, Roll JB, Osswald H, Kloor D: Hyperhomocysteinemia is associated with decreased erythropoietin expression in rats. Cell Physiol Biochem 2010;26:449-456.

4 Li CX, Xia M, Han WQ, Li XX, Zhang C, Boini KM, Liu XC, Li PL: Reversal by growth hormone of homocysteine-induced epithelial-to-mesenchymal transition through membrane raft-redox signaling in podocytes. Cell Physiol Biochem 2011;27:691-702.

5 Yi F, Chen QZ, Jin S, Li PL: Mechanism of homocysteine-induced rac1/nadph oxidase activation in mesangial cells: Role of guanine nucleotide exchange factor vav2. Cell Physiol Biochem 2007;20:909-918.

6 Austin RC, Lentz SR, Werstuck GH: Role of hyperhomocysteinemia in endothelial dysfunction and atherothrombotic disease. Cell Death Differ 2004;11:S56-64.

7 Cavalca V, Cighetti G, Bamonti F, Loaldi A, Bortone L, Novembrino C, De Franceschi M, Belardinelli R, Guazzi MD: Oxidative stress and homocysteine in coronary artery disease. Clin Chem 2001;47:887-892.

8 Ientile R, Curro M, Ferlazzo N, Condello S, Caccamo D, Pisani F: Homocysteine, vitamin determinants and neurological diseases. Front Biosci (Schol Ed) 2010;2:359-372.

-9 Robinson K, Gupta A, Dennis V, Arheart K, Chaudhary D, Green R, Vigo P, Mayer EL, Selhub J, Kutner M, Jacobsen DW: Hyperhomocysteinemia confers an independent increased risk of atherosclerosis in endstage renal disease and is closely linked to plasma folate and pyridoxine concentrations. Circulation 1996;94:2743-2748.

10 Abais JM, Xia M, Li G, Gehr TW, Boini KM, Li PL: Contribution of endogenously produced reactive oxygen species to the activation of podocyte nlrp3 inflammasomes in hyperhomocysteinemia. Free Radic Biol Med 2014;67:211-220.

11 Abais JM, Zhang C, Xia M, Liu Q, Gehr T, Boini KM, Li PL: Nadph oxidase-mediated triggering of inflammasome activation in mouse podocytes and glomeruli during hyperhomocysteinemia. Antioxid Redox Signal 2013;18:1537-1548.

12 Sen U, Munjal C, Qipshidze N, Abe O, Gargoum R, Tyagi SC: Hydrogen sulfide regulates homocysteinemediated glomerulosclerosis. Am J Nephrol 2010;31:442-455.

13 Zhang C, Boini KM, Xia M, Abais JM, Li X, Liu Q, Li PL: Activation of nod-like receptor protein 3 inflammasomes turns on podocyte injury and glomerular sclerosis in hyperhomocysteinemia. Hypertension 2012;60:154-162.

14 Li N, Chen L, Muh RW, Li PL: Hyperhomocysteinemia associated with decreased renal transsulfuration activity in dahl s rats. Hypertension 2006;47:1094-1100.

15 Mariathasan S, Newton K, Monack DM, Vucic D, French DM, Lee WP, Roose-Girma M, Erickson S, Dixit VM: Differential activation of the inflammasome by caspase-1 adaptors asc and ipaf. Nature 2004;430:213-218.

16 Anders HJ, Muruve DA: The inflammasomes in kidney disease. J Am Soc Nephrol 2011;22:1007-1018.

17 Vilaysane A, Chun J, Seamone ME, Wang W, Chin R, Hirota S, Li Y, Clark SA, Tschopp J, Trpkov K, Hemmelgarn BR, Beck PL, Muruve DA: The nlrp3 inflammasome promotes renal inflammation and contributes to ckd. J Am Soc Nephrol 2010;21:1732-1744.

18 Martinon F, Mayor A, Tschopp J: The inflammasomes: Guardians of the body. Annu Rev Immunol 2009;27:229-265. 


\section{Cellular Physiology and Biochemistry}

Cell Physiol Biochem 2014;34:829-841

\begin{tabular}{l|l}
\hline DOI: $10.1159 / 000363046$ & (c) 2014 S. Karger AG, Basel
\end{tabular}

Xia et al.: Nlrp3 in hHcys-Induced Inflammasome Activation

19 Gross 0, Thomas CJ, Guarda G, Tschopp J: The inflammasome: An integrated view. Immunolog rev 2011;243:136-151.

20 Tschopp J, Schroder K: Nlrp3 inflammasome activation: The convergence of multiple signalling pathways on ros production? Nat Rev Immunol 2010;10:210-215.

-21 Zhou R, Tardivel A, Thorens B, Choi I, Tschopp J: Thioredoxin-interacting protein links oxidative stress to inflammasome activation. Nat Immunol 2010;11:136-140.

-22 Cruz CM, Rinna A, Forman HJ, Ventura AL, Persechini PM, Ojcius DM: Atp activates a reactive oxygen species-dependent oxidative stress response and secretion of proinflammatory cytokines in macrophages. J Biol Chem 2007;282:2871-2879.

-23 Halle A, Hornung V, Petzold GC, Stewart CR, Monks BG, Reinheckel T, Fitzgerald KA, Latz E, Moore KJ, Golenbock DT: The nalp3 inflammasome is involved in the innate immune response to amyloid-beta. Nat Immunol 2008;9:857-865.

-24 Nour AM, Yeung YG, Santambrogio L, Boyden ED, Stanley ER, Brojatsch J: Anthrax lethal toxin triggers the formation of a membrane-associated inflammasome complex in murine macrophages. Infect Immun 2009;77:1262-1271.

-25 Martinon F, Burns K, Tschopp J: The inflammasome: A molecular platform triggering activation of inflammatory caspases and processing of proil-beta. Mol Cell 2002;10:417-426.

-26 Srinivasula SM, Poyet JL, Razmara M, Datta P, Zhang Z, Alnemri ES: The pyrin-card protein asc is an activating adaptor for caspase-1. J Biol Chem 2002;277:21119-21122.

27 Aganna E, Martinon F, Hawkins PN, Ross JB, Swan DC, Booth DR, Lachmann HJ, Bybee A, Gaudet R, Woo P, Feighery C, Cotter FE, Thome M, Hitman GA, Tschopp J, McDermott MF: Association of mutations in the nalp3/cias1/pypaf1 gene with a broad phenotype including recurrent fever, cold sensitivity, sensorineural deafness, and aa amyloidosis. Arthritis Rheum 2002;46:2445-2452.

28 Agostini L, Martinon F, Burns K, McDermott MF, Hawkins PN, Tschopp J: Nalp3 forms an il-1betaprocessing inflammasome with increased activity in muckle-wells autoinflammatory disorder. Immunity 2004;20:319-325.

29 Dostert C, Petrilli V, Van Bruggen R, Steele C, Mossman BT, Tschopp J: Innate immune activation through nalp3 inflammasome sensing of asbestos and silica. Science 2008;320:674-677.

-30 Imaeda AB, Watanabe A, Sohail MA, Mahmood S, Mohamadnejad M, Sutterwala FS, Flavell RA, Mehal WZ: Acetaminophen-induced hepatotoxicity in mice is dependent on tlr9 and the nalp3 inflammasome. J Clin Invest 2009;119:305-314.

-31 Martinon F, Petrilli V, Mayor A, Tardivel A, Tschopp J: Gout-associated uric acid crystals activate the nalp3 inflammasome. Nature 2006;440:237-241.

32 Menu P, Pellegrin M, Aubert JF, Bouzourene K, Tardivel A, Mazzolai L, Tschopp J: Atherosclerosis in apoedeficient mice progresses independently of the nlrp3 inflammasome. Cell Death Dis 2011;2:e137.

-33 Freigang S, Ampenberger F, Spohn G, Heer S, Shamshiev AT, Kisielow J, Hersberger M, Yamamoto M, Bachmann MF, Kopf M: Nrf2 is essential for cholesterol crystal-induced inflammasome activation and exacerbation of atherosclerosis. Eur J Immunol 2011;41:2040-2051.

-34 Rajamaki K, Lappalainen J, Oorni K, Valimaki E, Matikainen S, Kovanen PT, Eklund KK: Cholesterol crystals activate the nlrp3 inflammasome in human macrophages: A novel link between cholesterol metabolism and inflammation. PLoS One 2010;5:e11765.

-35 Salminen A, Ojala J, Suuronen T, Kaarniranta K, Kauppinen A: Amyloid-beta oligomers set fire to inflammasomes and induce alzheimer's pathology. J Cell Mol Med 2008;12:2255-2262.

-36 Lewis TL, Cao D, Lu H, Mans RA, Su YR, Jungbauer L, Linton MF, Fazio S, LaDu MJ, Li L: Overexpression of human apolipoprotein a-i preserves cognitive function and attenuates neuroinflammation and cerebral amyloid angiopathy in a mouse model of alzheimer disease. J Biol Chem 2010;285:36958-36968.

-37 Savage CD, Lopez-Castejon G, Denes A, Brough D: Nlrp3-inflammasome activating damps stimulate an inflammatory response in glia in the absence of priming which contributes to brain inflammation after injury. Front Immunol 2012;3:288.

-38 Rosin DL, Okusa MD: Dangers within: Damp responses to damage and cell death in kidney disease. J Am Soc Nephrol 2011;22:416-425.

-39 Vasileiou E, Montero RM, Turner CM, Vergoulas G: P2x(7) receptor at the heart of disease. Hippokratia 2010;14:155-163. 


\section{Cellular Physiology and Biochemistry}

Cell Physiol Biochem 2014;34:829-841

\begin{tabular}{l|l}
\hline DOI: $10.1159 / 000363046$ & (c) 2014 S. Karger AG, Basel
\end{tabular}

Xia et al.: NIrp3 in hHcys-Induced Inflammasome Activation

40 Boini KM, Xia M, Abais JM, Li G, Pitzer AL, Gehr TW, Zhang Y, Li PL: Activation of inflammasomes in podocyte injury of mice on the high fat diet: Effects of asc gene deletion and silencing. Biochim Biophys Acta 2014;1843:836-845.

-41 Iyer SS, Pulskens WP, Sadler JJ, Butter LM, Teske GJ, Ulland TK, Eisenbarth SC, Florquin S, Flavell RA, Leemans JC, Sutterwala FS: Necrotic cells trigger a sterile inflammatory response through the nlrp3 inflammasome. Proc Natl Acad Sci U S A 2009;106:20388-20393.

42 Boini KM, Xia M, Xiong J, Li C, Payne LP, Li PL: Implication of cd38 gene in podocyte epithelial-tomesenchymal transition and glomerular sclerosis. J Cell Mol Med 2012;16:1674-1685.

43 Chen YF, Li PL, Zou AP: Effect of hyperhomocysteinemia on plasma or tissue adenosine levels and renal function. Circulation 2002;106:1275-1281.

44 Zhang C, Hu JJ, Xia M, Boini KM, Brimson CA, Laperle LA, Li PL: Protection of podocytes from hyperhomocysteinemia-induced injury by deletion of the gp91phox gene. Free Radic Biol Med 2010;48:1109-1117.

-45 Boini KM, Zhang C, Xia M, Poklis JL, Li PL: Role of sphingolipid mediator ceramide in obesity and renal injury in mice fed a high-fat diet. J Pharmacol Exp Ther 2010;334:839-846.

46 Boini KM, Amann K, Kempe D, Alessi DR, Lang F: Proteinuria in mice expressing pkb/sgk-resistant gsk3. Am J Physiol Renal Physiol 2009;296:F153-159.

47 Watanabe H, Gaide O, Petrilli V, Martinon F, Contassot E, Roques S, Kummer JA, Tschopp J, French LE: Activation of the il-1beta-processing inflammasome is involved in contact hypersensitivity. J Invest Dermatol 2007;127:1956-1963.

48 Nishi Y, Satoh M, Nagasu H, Kadoya H, Ihoriya C, Kidokoro K, Sasaki T, Kashihara N: Selective estrogen receptor modulation attenuates proteinuria-induced renal tubular damage by modulating mitochondrial oxidative status. Kidney Int 2013;83:662-673.

-49 Wang C, Pan Y, Zhang QY, Wang FM, Kong LD: Quercetin and allopurinol ameliorate kidney injury in stztreated rats with regulation of renal nlrp3 inflammasome activation and lipid accumulation. PLoS One 2012;7:e38285.

50 Wang W, Wang X, Chun J, Vilaysane A, Clark S, French G, Bracey NA, Trpkov K, Bonni S, Duff HJ, Beck PL, Muruve DA: Inflammasome-independent nlrp3 augments tgf-beta signaling in kidney epithelium. J Immunol 2013;190:1239-1249.

-51 Dressler GR: The cellular basis of kidney development. Annu Rev Cell Dev Biol 2006;22:509-529.

-52 Hou CC, Wang W, Huang XR, Fu P, Chen TH, Sheikh-Hamad D, Lan HY: Ultrasound-microbubble-mediated gene transfer of inducible smad7 blocks transforming growth factor-beta signaling and fibrosis in rat remnant kidney. Am J Pathol 2005;166:761-771.

-53 Koike H, Tomita N, Azuma H, Taniyama Y, Yamasaki K, Kunugiza Y, Tachibana K, Ogihara T, Morishita R: An efficient gene transfer method mediated by ultrasound and microbubbles into the kidney. J Gene Med 2005; 7:108-116.

54 Lan HY, Mu W, Tomita N, Huang XR, Li JH, Zhu HJ, Morishita R, Johnson RJ: Inhibition of renal fibrosis by gene transfer of inducible smad7 using ultrasound-microbubble system in rat uuo model. J Am Soc Nephrol 2003;14:1535-1548.

-55 Sheyn D, Kimelman-Bleich N, Pelled G, Zilberman Y, Gazit D, Gazit Z: Ultrasound-based nonviral gene delivery induces bone formation in vivo. Gene Ther 2008;15:257-266.

-56 Bekeredjian R, Chen S, Frenkel PA, Grayburn PA, Shohet RV: Ultrasound-targeted microbubble destruction can repeatedly direct highly specific plasmid expression to the heart. Circulation 2003;108:1022-1026.

-57 Tsunoda S, Mazda O, Oda Y, Iida Y, Akabame S, Kishida T, Shin-Ya M, Asada H, Gojo S, Imanishi J, Matsubara $\mathrm{H}$, Yoshikawa T: Sonoporation using microbubble br14 promotes pdna/sirna transduction to murine heart. Biochem Biophys Res Commun 2005;336:118-127. 